\section{$\underset{\substack{\text { hommes } \\ \text { \& migrations }}}{ }$}

\section{Hommes \& migrations}

Revue française de référence sur les dynamiques

migratoires

\section{$1299 \mid 2012$}

Musulmanes et féministes en Grande-Bretagne

\title{
Les points aveugles
}

\section{Mustapha Harzoune}

\section{OpenEdition \\ Journals}

Édition électronique

URL : http://journals.openedition.org/hommesmigrations/1868

DOI : 10.4000/hommesmigrations.1868

ISSN : 2262-3353

\section{Éditeur}

Musée national de l'histoire de l'immigration

\section{Édition imprimée}

Date de publication : 1 septembre 2012

Pagination : 134-141

ISSN : 1142-852X

\section{Référence électronique}

Mustapha Harzoune, "Les points aveugles », Hommes \& migrations [En ligne], 1299 | 2012, mis en ligne le 29 mai 2013, consulté le 22 septembre 2020. URL : http://journals.openedition.org/

hommesmigrations/1868; DOI : https://doi.org/10.4000/hommesmigrations.1868

Ce document a été généré automatiquement le 22 septembre 2020.

Tous droits réservés 


\title{
Les points aveugles
}

\author{
Mustapha Harzoune
}

1 Henri Lopes dans Une enfant de Poto-Poto (Gallimard, 2012, prix littéraire 2012 de la Porte Dorée) fait dire à un de ses personnages, que le métis "nous a appris à devenir des êtres humains. D'ici et d'ailleurs". Au point que la notion même "dépérira. Dans quelques décennies, peut-être avant un siècle, il n'y aura plus de métis, mais des Français, des Congolais, des Sénégalais, des Américains, blancs, noirs, bruns... Les 'pur-sang' n'oseront plus se vanter de ce qui deviendra une tare". Voilà de quoi affoler les gardiens de l'ordre identitaire. Cela est même inconcevable pour une grande partie de nos contemporains. Amin Maalouf, dans son dernier roman (Les Désorientés, Grasset, 2012), évoque lui ces "points aveugles", ces "aspects de la réalité", "inconcevables", "invisibles" aux hommes de leur temps et qui, quelques décennies plus tard, apparaissent comme "évidents". En matière d'identité, de repères géographiques, d'appartenance nationale..., les vieux totems sont peut-être en train de laisser un peu de place à de nouvelles représentations, de nouveaux imaginaires. Des points encore aveugles semblent s'esquisser à l'horizon des regards contemporains. Quid en matière de migrations ? Les migrants seront-ils encore demain réduits à une variable d'ajustement, à des corps inertes avec lesquels on pourrait jouer selon son bon plaisir et ses besoins? "Mamadou m'a dit, Mamadou m'a dit: 'On a pressé le citron, on peut jeter la peau"', chantait en 1979 - déjà - François Béranger... L'incapacité à saisir en quoi le migrant est un acteur économique doué de raison et de raisonnement, dont les actes et les projets sont guidés par une étude rationnelle des coûts et avantages d'un investissement qui peut parfois se payer cash, ne serait-il pas, ici, un de ces "points aveugles" ? Comme d'admettre que la migration est une variable économique et démographique incontournable non seulement à l'échelle de chaque pays mais aussi des continents, et plutôt que de la représenter comme un épouvantail et une maladie, ne serait-il pas plus judicieux de l'intégrer dans les têtes et dans les cœurs comme une partie intégrante de la marche des nations de plus en plus diverses et d'un monde globalisé ? Ce qui apparaît comme "évident" pour certains est loin de l'être pour d'autres.

2 Ainsi, les déclarations de Cecilia Malmström, selon qui "l'immigration sera nécessaire pour l'Europe", ont provoqué le courroux de nombre de commentateurs (Le Monde, 10 juillet 2012). Pour la commissaire européenne aux Affaires intérieures, "l'immigration sera 
nécessaire, étant donné notamment l'évolution de la démographie dans la plupart de nos pays. On estime qu'en 2030, sans nouvelle immigration, la population européenne en âge de travailler aura diminué de $12 \%$. Des chefs d'entreprise me confient régulièrement leurs difficultés à recruter des gens qualifiés alors même que le chômage est au plus haut. L'Allemagne manque d'ingénieurs; d'ici à 2020, le secteur européen de la santé devrait manquer de 2 millions de personnes. Or un rapport de l'OCDE vient de montrer que beaucoup de personnes susceptibles d'émigrer sont moins tentées par l'Europe et davantage par le Brésil, le Canada ou l'Australie, voire l'Angola ou le Mozambique. Cela pourrait, à terme, nous causer de grosses difficultés. La même OCDE montre d'ailleurs que la pression migratoire sur l'Europe s'allège. Mais, bien sûr, ce peut être un effet temporaire de la crise que nous connaissons". Pour Cecilia Malmström, "il ne s'agit pas d'ouvrir les portes en grand, mais de s'interroger sur la façon de faciliter une nouvelle immigration légale - ce qui est d'ailleurs aussi une manière de lutter contre l'immigration illégale".

3 Par ailleurs, en matière d'intégration, elle constate "un échec", "dans la plupart des grandes villes (...). La ghettoïsation est une réalité dont les politiques sont responsables parce qu'ils n'ont pas agi avec suffisamment de vigueur". À ce propos quelques rappels et repères ne sont pas inutiles. Le 18 juillet, le site de l'Alsace.fr rappelait que "la politique de la Ville, à l'œuvre depuis une vingtaine d'années, vise 751 zones urbaines sensibles (ZUS) qui abritent 4,4 millions d'habitants. Selon l'Observatoire national des ZUS, plus d'un habitant sur deux y est immigré ou descendant d'immigré. Près d'un immigré sur cinq et près d'un descendant d'immigré sur sept vivent en ZUS contre une personne sur quinze sur l'ensemble de la population métropolitaine. Dans les ZUS de la région parisienne, le pourcentage de personnes issues de l'immigration monte à $64 \%$. En 2010, le taux de chômage a progressé de 2,3 points par rapport à 2009, atteignant $20,9 \%$ : plus d'un actif sur cinq de 15 à 59 ans est ainsi au chômage, soit le taux le plus élevé depuis 2003. La moitié des habitants des ZUS ne dispose d'aucun diplôme supérieur au brevet des collèges contre un tiers dans les zones environnantes. En 2009, la part des personnes y vivant sous le seuil de la pauvreté (954 euros mensuels) était de $32,4 \%$, un taux deux fois plus élevé que celui de leur agglomération".

\section{Les vieilles certitudes}

4 “Comment peut-on proférer autant d'âneries! fulmine Robert Ménard. C'était mercredi dernier, dans le journal Le Monde, une interview de la commissaire européenne aux affaires intérieures, la Suédoise Cecilia Malmström. Et que nous dit cette brave dame? Tous les poncifs possibles et imaginables sur les bienfaits de l'immigration" (Site medialibre.eu, le 16 juillet). "Tout y est", écrit-il, et de reprendre, sans aucune démonstration, les propos de Cecilia Malmström. À la fin de son billet, il cède, lui, à d'autres "poncifs": "Mais qui fréquente-telle en dehors de ses collègues de la bureaucratie bruxelloise? Lui arrive-t-il de quitter la rue de la Loi et d'emprunter autre chose que la station de métro Schuman? Mais que lit-elle, elle qui explique que 'les milieux académiques considèrent d'ailleurs que ce que je dis est parfaitement banal'? Je lui propose pour ces vacances d'été un petit stage dans certains quartiers de nos grandes villes et la lecture du livre Les Yeux grands fermés de la démographe Michèle Tribalat."

5 À vrai dire il n'y a pas grand-chose dans le coup de gueule habituel de Ménard à se mettre sous la dent. Laurent Pinsolle, porte-parole du parti Debout la République, avance, lui, quelques arguments dans un papier mis en ligne le 17juillet sur 
Marianne2.fr. Pour limiter les flux migratoires et contester la commissaire européenne, il écrit que "tout d'abord, il faudrait lui rappeler que le taux de chômage vient de battre un record, à $11 \%$ de la population active. (...) Pire, l'OIT [l'Organisation internationale du travail] estime que nous risquons d'ajouter 4,5 millions de chômeurs aux 17,4 millions déjà recensés dans les quatre prochaines années ["si des politiques économiques favorisant la croissance et l'emploi ne sont pas engagées", dixit l'OIT]. Bref, les pays européens ont surtout besoin d'emplois aujourd'hui, bien plus que de nouveaux immigrés qui auraient toutes les difficultés à trouver un emploi. Et si le secteur de la santé risque de manquer de 2 millions de personnes en 2020, autant former des chômeurs ou des jeunes. Nous avons huit années pour le faire!" Ainsi, les emplois constitueraient un volume global, un bloc indifférencié, et les immigrés continueraient à affluer quelles que soient les tendances du marché du travail, quitte à se retrouver au chômage.

Le deuxième argument porte sur les différences démographiques: "Si l'Allemagne et l'Italie vont connaître un vrai krach démographique, les populations actives de France et du Royaume-Uni vont continuer à progresser, d'où des besoins différents." Sur ce point les prévisions de l'Insee diffèrent. En effet, la situation démographique française est généralement saluée comme meilleure, comparée à son voisin allemand, pour autant, pour l'Insee, le simple maintien de la population active en France à 34 millions de personnes en 2050 (pour 33 millions en 2012) exigerait de recourir à un flux de 150000 entrées d'immigrés par an (Insee, Projection de population active 2010-2060)

$7 \mathrm{Si}$, pour Laurent Pinsolle, les perspectives en matière d'emplois interdisent toute migration nouvelle, pour Ivan Rioufol, c'est l'identité qui serait en danger. Sur son blog, le 11 juillet, le chroniqueur du Figaro parle d'“aveuglement" et de "capitulation": " L'argumentaire technocratique, productif et comptable de Cecilia Malmström, persuadée que les citoyens européens pensent comme elle, exprime parfaitement l'aveuglement des élites face au multiculturalisme. Celui-ci est pourtant en train de bouleverser les équilibres identitaires et d'attiser les tensions au coeur de la plupart des nations ouvertes. Elle ne fait aucune référence aux notions de civilisations, de cultures, de peuples, de langues, de préservation des acquis historiques. En fait, l'Europe est vue par elle comme un immense hôtel, un hall d'aéroport où chacun aurait sa place en fonction de sa seule utilité technique, économique, reproductrice. C'est un monde plat, indifférencié et inhumain que défend, avec sa règle à calcul, ses statistiques et ses certitudes, Madame la commissaire. L'histoire retiendra la capitulation des responsables européens devant l'immigration."

8 Faire de l'immigration un bouc émissaire utile, agiter le chiffon rouge de la peur pour mieux dédouaner les autorités de leurs responsabilités, éviter l'effort consistant à rendre compte de la complexité et de la diversité des situations et des sujets, ne pas bousculer les vieux paradigmes et schémas, n'est pas l'apanage des seuls dirigeants et commentateurs du riche continent européen. Au Maroc, le pays qui se targue par ailleurs d'une forte communauté à l'étranger, l'immigration est, là aussi, "coupable selon le ministre de l'Emploi" (yabiladi.com, le 8 juillet): "Mai dernier, le Haut-Commissariat au Plan a fait état de la hausse du chômage au Maroc durant le $1^{\text {er }}$ trimestre de cette année par rapport à la même période en 2011. Officiellement $10 \%$ des Marocains sont sans emploi. Le gouvernement Benkirane a pris acte de cette augmentation et il semble qu'il est sur le point de s'attaquer sur l'origine de la crise du marché du travail au Maroc : l'immigration." "Les propos du ministre sont parfaitement en phase avec ceux de certains députés, à l'image d'Abdelhadi Khairat de l'USFP (opposition) et d'officiels accusant, notamment, des Subsahariens d'être des membres de réseaux terroristes en provenance du Sahel, de trafic de drogue ou de délinquance." 
Autre lieu, même schéma idéologique et politique. "L'ennemi de la vérité n'est pas le mensonge, mais les certitudes", disait Nietzsche.

Pourtant, tout le monde revendique et se réclame du principe de réalité et condamne l'idéologie chez l'autre, l'interlocuteur ou l'opposant. "Est-il possible en France de sortir de l'idéologie pour se concentrer sur la réalité ?" demande Maxime Tandonnet, haut fonctionnaire, ci-devant conseiller de Nicolas Sarkozy, notamment sur les questions de l'immigration (atlantico.fr, 29 juillet). Après un autosatisfecit sur l'"excellente politique, la meilleure politique possible destinée à contenir le flux de population", de la présidence Sarkozy (on est jamais mieux servit que par soi-même), il écrit : "À mes yeux (et j'aimerais tant me tromper), les flux migratoires à vocation de peuplement, d'un niveau déjà élevé, sans doute aux alentours de 150000 à 200000 par an, ont toute chance d'augmenter à l'avenir dans des proportions significatives. Dans un pays qui compte trois millions de chômeurs et n'a pas d'emploi à proposer aux nouveaux arrivants, ni d'ailleurs de logement, et dont la situation budgétaire interdit de consacrer les moyens considérables nécessaires à l'intégration des primoarrivants, ces mouvements de population vont encore amplifier l'exclusion, le repli, la ghettoïsation, le sentiment de révolte et les phénomènes de chaos et d'anomie tels que les a décrits le sociologue Hugues Lagrange. (...)

Sortir de l'idéologie et des passions, à droite comme à gauche, pour se focaliser sur les seules réalités: est-ce encore possible dans notre pays?" Ménard enrôlait Michèle Tribalat, Tandonnet se charge lui de Hugues Lagrange... Une autre question se pose alors : est-il possible de citer sans déformer, sans réduire, sans simplifier?

\section{Ailleurs, en Europe}

11 Ailleurs, en l'occurrence au Royaume-Uni, l'OBR (le Bureau de responsabilité budgétaire), "estime que l'arrivée de main-d'œuvre étrangère pourrait non seulement faire croître les recettes fiscales, mais aussi relancer l'économie. 'Plus d'immigration pourrait permettre au Royaume-Uni de faire passer ses prévisions de croissance annuelle de $2,4 \%$ à $2,7 \%$ sur les cinquante prochaines années'. Selon l'OBR, le vieillissement de la population britannique pourrait générer un manque à gagner pour les recettes fiscales de plus de 17 milliards de livres, soit près de 21 milliards d'euros. Si Westminster décide de fermer les portes du pays pendant les cinquante prochaines années, le Royaume-Uni perdrait 0,4 point de croissance et la dette publique grimperait en flèche pour atteindre plus de $120 \%$ du PIB. D'après les statistiques du Parlement, un Britannique sur quatre aura soixante ans ou plus en 2050 , contre seulement un sur six à l'heure actuelle. 'Maintenir, voire augmenter le nombre annuel d'immigrés, soulagerait notre emprunt et la dette publique, car les nouveaux venus ont plus de chances d'être en âge de travailler que la population [vieillissante]', note le Bureau pour la responsabilité budgétaire" (The Independant, 13 juillet, repris par myeurope.info, 13 juillet, et www.terraeco.net, 16 juillet).

12 En Allemagne, la situation démographique (1,4 enfant par femme) inquiète aussi, et les besoins en immigrés pour soutenir l'activité économique et les comptes sociaux sont tels que Berlin a ouvert un site très suggestif pour faire venir à lui les petits surdoués des migrations internationales: www.make-it-in-germany.com/ (La Croix, 2 juillet). Le 5 juillet, le site d'Europe 1 rapporte qu'“en avril, la Bundesbank a calculé que l'Allemagne avait besoin de 200000 immigrés supplémentaires par an pour soutenir la croissance de la production économique. 'En 2025, il manquera à l'Allemagne, pour des raisons purement 
démographiques, jusqu'à six millions de personnes en âge de travailler', a fait savoir début juin la ministre du Travail Ursula von der Leyen. Depuis avril dernier, le gouvernement a d'ailleurs simplifié les conditions d'installation en Allemagne pour les étudiants étrangers. Ainsi, les titulaires d'un diplôme supérieur ou de cinq ans d'expérience professionnelle bénéficieront désormais [...] de la 'carte bleue', titre de séjour spécifiquement destiné aux étrangers qualifiés, dès qu'ils disposent d'un contrat de travail assurant un revenu de 44800 euros par an". “ L'Allemagne attire déjà, depuis quelques mois, de plus en plus de jeunes diplômés en provenance des pays d'Europe du Sud en récession, qui manquent de perspectives d'avenir dans leur pays. Le nombre d'immigrés en provenance de Grèce l'an dernier a ainsi progressé de $90 \%$ par rapport à 2010, tandis que le nombre des nouveaux arrivants venus d'Espagne grimpait de $52 \%$ en un an, selon des chiffres publiés récemment."

Le 25 juillet, le site du Nouvel Obs indiquait que "la population allemande a augmenté en 2011, pour la première fois en dix ans, grâce à l'afflux de populations immigrées venues d'Europe de l'Est qui a compensé le plus bas taux de natalité enregistré depuis l'après-guerre. 'Tandis que l'immigration des pays durement touchés par la crise (de la zone euro) comme la Grèce et l'Espagne a augmenté, le moteur de cette hausse (...) sont les pays du bloc de l'Est qui ont rejoint l'Union européenne en 2004 et 2007', a déclaré à Reuters la porte-parole de l'organisme." Il s'agit de Polonais (163000 en 2011), de Hongrois (41000) de Bulgares et de Roumains (146000). "Cet afflux de populations immigrées vers la première économie d'Europe a permis de compenser la baisse des naissances. L'Allemagne a enregistré 663000 naissances en 2011, contre 678000 en 2010. Le nombre de décès a dépassé les naissances, comme chaque année depuis 1972. La différence était de 190000 en 2011."

Mais attention, "si l'Allemagne a besoin de travailleurs qualifiés, notamment de docteurs et ingénieurs, l'entreprise peut s'avérer plus hasardeuse pour les autres travailleurs" (La Tribune.fr, le 9 juillet). Ainsi, "comme 23800 Grecs et 20700 Espagnols en 2011 - des effectifs en hausse de respectivement $90 \%$ et $52 \%$ par rapport à 2010 -, [Alejandro] était venu tenter sa chance en Allemagne au printemps 2011. En poche, son diplôme de commerce international." Mais voilà, "sans diplôme dans le secteur de la santé ou des techniques comme l'ingénierie ou l'informatique, où il $\mathrm{y}$ a de très gros besoins, il est très difficile de trouver un emploi en Allemagne. On entre alors en compétition avec les Allemands. "Et si en plus, comme Alejandro, on ne parle pas l'allemand...

Pour autant, "une étude publiée début juillet par l'institut IW Cologne souligne la progression des diplômés parmi les immigrés en Allemagne, avec $27,5 \%$ des travailleurs titulaires d'un diplôme de l'enseignement supérieur ces dix dernières années, contre $18 \%$ des Allemands".

\section{Les pépins de la réalité}

De manière plus large, et selon le rapport de l'OCDE Perspectives sur les migrations internationales 2012" "si l'OCDE vieillissante entend continuer à croître, elle doit d'urgence mettre sur pied une politique volontariste d'immigration" (Slate.fr, 2 juillet). Le rapport note que "dans l'OCDE, les flux migratoires ont baissé pendant la crise. L'immigration permanente à destination des pays membres a diminué de 2,5\% en 2010, avec 4.1 millions de personnes concernées : $-8 \%$ aux États-Unis, $-3 \%$ pour les pays européens - sans tenir compte des mouvements intra-européens. Et les politiques n'y 
sont pas pour grand-chose. Ainsi, note l'organisation : 'Au Royaume-Uni, l'an dernier, en dépit d'une limitation drastique de la migration hautement qualifiée, les employeurs ont si peu embauché que les limites imposées n'ont jamais été atteintes.' 'Aux ÉtatsUnis, avant la crise, les quotas de visas H-1B pour les travailleurs qualifiés étaient souvent comblés en quelques jours. En 2009 et 2010, il a fallu plus de neuf mois'."

Et ce phénomène ne concernerait pas seulement l'immigration légale, car l'immigration illégale diminuerait aussi lorsque les temps sont durs: "En effet, ces mouvements ne sont pas sans coût et, sans aucune garantie d'emploi, ils deviennent moins attrayants pour les migrants potentiels et leurs familles." Ainsi, aux États-Unis, les appréhensions aux frontières ont baissé de 875000 en 2007 à 350000 en 2011.

France et ses $+8 \%$ de flux d'immigration permanente entre 2009 et 2010 infirmeraitelle la corrélation constatée entre flux migratoires et santé du marché du travail ? Non, car selon l'OCDE, 'l'essentiel des 'nouveaux' migrants de 2010 résidaient déjà dans le pays en 2009, mais n'ont obtenu le statut d'immigrés 'permanents' qu'en 2010".

Les pays en croissance, en revanche, ont continué de voir leurs flux migratoires se gonfler : $+10 \%$ vers le Canada, la Corée et le Mexique. En 2011, la timide reprise dans l'OCDE a inversé les tendances : comme le note l'organisation, "la hausse a repris en 2011 en Australie, aux États-Unis et en Nouvelle-Zélande, ainsi que dans la plupart des pays européens de l'OCDE, à l'exception de l'Italie, de l'Espagne et de la Suède".

Selon l'OCDE, libéraliser les flux migratoires ne provoquerait pas des arrivées massives. En d'autres termes, les besoins du marché priment. Et de citer l'exemple de "l'introduction à la fin de l'année 2008 d'un régime migratoire libéral en Suède, [qui] a effectivement entraîné une demande accrue de la part des employeurs, mais cette demande n'a cependant pas explosé".

21 "Si les flux migratoires varient avec la conjoncture, pas question cependant d'en faire une variable d'ajustement. L'Espagne, qui avait employé beaucoup d'immigrés non qualifiés pour subvenir aux besoins du bâtiment - aujourd'hui en pleine déconfiture en sait quelque chose. Car les migrants sont les premiers touchés par le chômage, et tout particulièrement par le chômage de longue durée, surtout s'ils sont peu qualifiés. Ce qui ne contribue guère à faciliter leur intégration dans le pays... Mieux vaut donc mener une politique d'immigration volontariste et sur le long terme en veillant à la bonne intégration des personnes migrantes, conseille l'OCDE. Car Angel Gurria [secrétaire général de l'OCDE] n'a pas peur de l'affirmer: l'immigration est indispensable aux économies occidentales! Qu'on en juge : sur les dix dernières années, les nouveaux migrants ont contribué à hauteur de $70 \%$ à l'augmentation de la population active en Europe et à $47 \%$ aux États-Unis. 'Mais les flux migratoires actuels ne suffiront pas pour maintenir à son niveau actuel la population en âge de travailler dans de nombreux pays, particulièrement en Europe', prévient l'OCDE."

Sur le plan démographique, il faudrait même s'inquiéter de la pérennité de telle ou telle nation. Ainsi, Courrier international (18 juillet) titre sur cette question: "Les Portugais, extinction prévue en 2204 ?" Rien moins ! Plus sérieusement, il est écrit que "le Portugal se vide de ses habitants. La population vieillit, les naissances déclinent et l'immigration freine. Sans parler de la crise qui pousse les jeunes diplômés à aller chercher un avenir meilleur ailleurs". Afrik.com, du 30 mai, rappelle que "pour fuir la misère dans leur pays, certains Européens n'hésitent plus à migrer vers l'Afrique en espérant trouver du travail". Et de citer les Espagnols qui s'établissent du côté d'Oran en Algérie et les Portugais qui s'esbignent 
vers l'Angola. "La presse portugaise n'a pas manqué de faire le constat de l'augmentation du nombre de migrants portugais en Angola. Selon un quotidien de Lisbonne, en 2006, seulement 156 visas de Portugais en partance vers l'Angola étaient recensés. En 2011, leur nombre a explosé, atteignant 23 787. En 2012, on compte près de 100000 Portugais résidant en Angola, soit le triple des Angolais installés au Portugal. Une migration que les autorités portugaises encourageraient, selon le journal camerounais Camer.be."

Tandis que les médias rappellent que l'Europe a besoin d'immigrés, que sur un continent vieillissant certains pays se décatissent, que l'Europe perdant de son attractivité, les flux migratoires pourraient se diriger vers d'autres destinations. Le 13 août, le site Atlantico rendait compte d'une étude signée par Constantin Gurdgiev, professeur de finance au Trinity College de Dublin. Il s'agit en fait d'un graphique présenté sur le TrueEconomics.blogspot.com et baptisé par son auteur le "terrifiant graphique de la décennie". Que dit ce graphique? Que si le taux de dépendance (rapport retraités sur population active) augmente en Europe, il a aussi tendance à augmenter dans les pays du Sud, de sorte que "l'occident" pourrait "manquer d'immigrés pour payer les retraites de ses personnes âgées": "vers 2030, les jeunes travailleurs immigrés qui fournissaient une force de travail bon marché aux nations développées vont commencer à devoir soutenir les personnes âgées restées dans leur pays d'origine". Selon Gurdgiev, deux scénarios sont alors envisageables, soit les immigrés dépenseront une large part de leur paie dans leur pays d'origine (ce qui est déjà en partie le cas avec les importants transferts d'argent qui servent à la consommation dans les pays d'origine), soit les flux d'émigration vers les pays développés se réduiront.

Cela va vraiment mal en Europe. Quelques-uns de ces "points aveugles" risquent de sauter à la figure : non seulement l'immigration pourrait bouder, d'ici à moins d'une génération, le vieux continent, mais, de plus, les flux pourraient même s'inverser : "Un éditorialiste portugais indiquait d'ailleurs récemment: 'La vieille Europe déprime. La zone euro est au bord de l'implosion et la récession menace. En Afrique, la croissance économique se maintient. Et si les jeunes Européens traversaient la Méditerranée pour émigrer?" (www.afrik.com, 30 mai). Un point encore aveugle qui a d'ailleurs fait l'objet d'un livre pour enfants (Janne Teller, Guerre. Et si ça nous arrivait?, Les Grandes personnes, 2012). Peut-être que les plus jeunes se montreront plus clairvoyants. 